Bull. Austral. Math. Soc.

$20 \mathrm{~F} 05,57 \mathrm{M} 20$

Vol. 50 (1994) [21-27]

\title{
GROUP EXTENSIONS ARE QUASI-SIMPLY-FILTRATED
}

\author{
S.G. BRick and M.L. Mihalik
}

A finitely presented group $G$ is quasi-simply-filtrated (abbreviated qsf) if, given a finite complex with fundamental group $G$, the universal cover of the complex can be "approximated" by simply connected finite complexes. This notion is a generalisation of a concept of Casson's used in the study of three-manifolds.

In this paper we show that any extension of a finitely presented infinite group by a finitely presented infinite group is qsf.

\section{INTRODUCTION}

Suppose $X$ is a finite complex. Write $\widetilde{X}$ for its universal cover. We say that $X$ is quasi-simply-filtrated (abbreviated qsf) if, given a connected finite subcomplex $C \subset \tilde{X}$, there is a simply connected finite complex $D$, and a cellular map $f: D \rightarrow \widetilde{X}$ with $f_{\mid f^{-1}(C)}: f^{-1}(C) \rightarrow C$ a homeomorphism.

In [2] it is shown that if $X_{1}$ and $X_{2}$ are finite complexes with isomorphic fundamental groups then $X_{1}$ is qsf if and only if $X_{2}$ is qsf. Thus one can say that a finitely presented group $G$ is qsf if some (and hence any) finite complex $X$, with $\pi_{1}(X)=G$, is qsf.

The qsf condition is developed in [2] (also see [5]) as a geometric generalisation of Andrew Casson's $C_{2}$ condition on presentations of groups (see [3]). Both of these conditions can be used to show that certain closed irreducible 3-manifolds are covered by $\mathbb{R}^{3}$. The advantage of the qsf condition, besides it being a geometric condition, is that it is an invariant of finitely presented groups whereas the validity of the $C_{2}$ condition may vary between different presentations of a group.

In [2] it is shown that all one-relator groups and all simply connected at infinity groups are qsf. And (see [2]) the qsf property is preserved by taking finite extensions, amalgamations, and HNN-extensions. In [4] all automatic and semihyperbolic groups are shown to be qsf.

The purpose of this paper is to expand the class of groups known to be qsf by proving the following result:

THEOREM. If $1 \rightarrow B \rightarrow G \rightarrow L \rightarrow 1$ is a short exact sequence of finitely presented infinite groups, then $G$ is qsf.

Received 13th September, 1993.

Copyright Clearance Centre, Inc. Serial-fee code: 0004-9729/94 \$A2.00+0.00. 


\section{Preliminaries}

Choose a finite presentation

$$
\mathcal{P}=\langle A \cup B \mid R \cup S \cup T\rangle
$$

of $G$ where $\langle A \mid R\rangle$ is a presentation of $H$ and each element of $T$ is of the form $b^{-1} a b w^{-1}$ where $a \in A^{ \pm 1}, b \in B^{ \pm 1}$ and $w=w(a, b)$ is some word in the generators A.

Take $X$ to be the two-complex associated to $\mathcal{P}$, and, as above, write $\tilde{X}$ for the universal cover of $X$. Let $Y$ be the two-complex associated to the presentation $\langle A \mid R\rangle$ of $H$. Then $Y \subset X$ and lying above $Y$ in $\tilde{X}$ is a collection of components, each component being a copy of the universal cover $\tilde{Y}$. There is one component for each coset of $H$ in $G$ or, equivalently, each element of $L$. Write $l \tilde{Y}$ for a typical component.

We shall refer to those edges of $\widetilde{X}$ lying above edges that correspond to elements of $A^{ \pm 1}$ as $A$-edges. An $A$-path or an $A$-loop is an edge path or loop consisting entirely of $A$-edges. We define $B$-edges in a similar fashion. And those two-cells of $\tilde{X}$ lying above two-cells corresponding to relators in $T$ will be called conjugation cells. Note that the attaching map of a typical conjugation cell is of the form $b_{1}^{-1} a b_{2} w$ where $b_{1}$ and $b_{2}$ are $B$-edges lying above the same edge in $X, a$ is an $A$-edge, and $w$ is an $A$-path lying above the path corresponding to the word $w=w(a, b)$ (thus we abuse notation slightly).

We shall work with subcomplexes of $\tilde{X}$ that are connected, finite and intersect each copy, $l \tilde{Y}$, of $\tilde{Y}$ in a connected (possibly empty) subset. Call such subcomplexes admissible.

Start with an arbitrary connected finite subcomplex $C \subset \tilde{X}$. Choose a basepoint * in $C$. By adding finitely many edges, we may assume that $C$ is admissible. We shall enlarge $C$ to a subcomplex $K$ by adjoining finitely many edges, and conjugation cells such that $\pi_{1}(K)$ can be killed by killing finitely many loops which are null-homotopic in $\widetilde{X} \backslash C$. Then we can attach disks to each such loop, yielding a simply connected finite complex $D$, and map each of the disks into $\tilde{X} \backslash C$. Clearly, this will suffice to show that $X$ is qsf.

So we need to study the generators of the fundamental group of finite subcomplexes. They are of two different types: conjugates of $A$-loops and other elements. Refer to those generators that are conjugates of $A$-loops as being of type 1 and the others as being of type 2 .

We need two lemmas on the effect of adjoining $A$-edges and conjugation cells.

Lemma 2.1. Suppose $Z$ is an admissible subcomplex of $\tilde{X}$ and $e$ is an A-edge that meets $Z$. Then $Z \cup e$ is admissible. And if $\pi_{1}(Z)$ is generated by $\left\{u_{1}, \ldots, u_{n}\right\}$ 
then $\pi_{1}(Z \cup e)$ is generated by $\left\{u_{1}, \ldots, u_{n}, \tau \lambda \tau^{-1}\right\}$, where $\lambda$ is an $A$-loop in the copy of $\tilde{Y}$ containing $e$.

Proof: We need only consider the case where both endpoints of $e$ lie in $Z$. Since $Z$ is admissible there is an $A$-path, $p$, in $Z$ from the terminal point of $e$ to the initial point of $e$. Let $\lambda=e p$. Take $\tau$ to be a path from the basepoint of $Z$ to the initial point of $e$. Now the result is immediate.

LEMмA 2.2. Suppose $Z$ is an admissible subcomplex of $\tilde{X}$ and $\Delta$ is a conjugation cell with $\partial \Delta=b_{1}^{-1} a b_{2} w^{-1}$, and $b_{1} \subset Z$. Then $Z \cup \Delta$ is admissible. Further if $\pi_{1}(Z)$ is generated by $\left\{u_{1}, \ldots, u_{n}\right\}$ then $\pi_{1}(Z \cup \Delta)$ is generated by $\left\{u_{1}, \ldots, u_{n}\right.$, $\left.v_{1}, \ldots, v_{m}\right\}$ where each $v_{i}$ is a type 1 generator. Moreover, if $a \subset Z$ then each $v_{i}$ is of the form $\tau \lambda \tau^{-1}$ where $\lambda$ is an A-loop in the copy of $\widetilde{Y}$ containing the endpoint of $b_{1}$.

ProOF: Applying lemma 2.1 repeatedly shows that $\pi_{1}(Z \cup a \cup w)$ is gotten from $\pi_{1}(Z)$ by adding some new type 1 generators.

If $a$ is in $Z$, then these new generators arise from adjoining $w$, and are of the desired form, again by lemma 2.1 .

If $b_{2}$ is not in $Z$ then $Z \cup a \cup w$ is a strong deformation retract of $Z \cup \Delta$. Otherwise $\pi_{1}(Z \cup \Delta)$ is obtained from $\pi_{1}(Z \cup a \cup w)$ by adding a new relator. In either case, the lemma follows.

For sake of brevity, we make the following convention: When we speak of adjoining conjugation cells to a subcomplex $Z$, we mean that $Z$ is admissible and that the cells may be indexed $\Delta_{1}, \Delta_{2}, \ldots, \Delta_{t}$ with attaching maps $\partial \Delta_{i}=b_{i 1}^{-1} a_{i} b_{i_{2}} w_{i}^{-1}$ such that $b_{11} \subset Z$ and $b_{i 1} \subset Z \cup \Delta_{1} \cup \cdots \cup \Delta_{i-1}$ for $i>1$.

The above lemmas show that if a generating set for $\pi_{1}(Z)$ has been chosen then adding finitely many $A$-edges or conjugation cells does not create any new type 2 generators. Hence if by adding such edges and cells we are able to homotope a single arbitrary type 2 generator to "nicer form" then by adding finitely many $A$-edges and conjugation cells, we can homotope all of the type 2 generators to such nicer forms.

\section{TyPe 2 genERATORS}

In this section we shall adjoin finitely many conjugation cells and $A$-edges to $C$, yielding an admissible subcomplex $C_{1}$ such that $\pi_{1}\left(C_{1}\right)$ has a set of generators with each type 2 generator being of the form $\delta_{1} \rho \delta_{2}$, where $\rho$ is a loop null-homotopic in $\widetilde{X} \backslash C$ and $\delta_{1} \delta_{2}$ is an $A$-loop.

Fix a generating set for $\pi_{1}(C)$. First observe that adjoining conjugation cells lets us move $B$-edges to the right of $A$-edges (where doing so replaces the $A$-edges with $A$-paths). See Figure 3.1. Note that the conjugation cell pictured lies above the cell in $X$ corresponding to the relator $\left(b^{-1}\right)^{-1} a b^{-1} w^{-1}$ where $w=w\left(a, b^{-1}\right)$. Hence we may 
assume, at the cost of adding more type 1 generators, that each type 2 generator is of the form $\alpha \beta$, where $\alpha$ is an $A$-path and $\beta$ is a $B$-path.

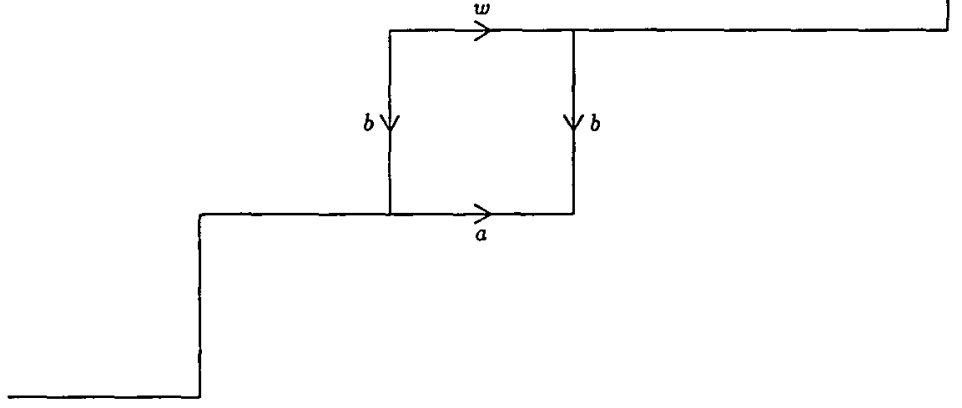

Figure 3.1

Let $m$ be the maximum of the lengths of the type 2 generators. By $[1$, lemma 3.1], there is an integer $N$ such that an edge loop in $\widetilde{X}$ based at a vertex $v$ and having length $\leqslant m$ is null-homotopic in the $N$-neighbourhood of $v$, that is, the iterated star (without subdivision), $\operatorname{star}^{N}(v)$.

Since $H$ is infinite, we can find an $A$-path, $\gamma$, from the basepoint $*$ to a vertex $v$ not in the $N$-neighbourhood of $C$. Observe that the $N$-neighbourhood of $v$ is disjoint from $C$.

Consider an arbitrary type 2 generator $\alpha \beta$. Write $\alpha^{\prime} \beta^{\prime}$ for the translate of $\alpha \beta$ to the vertex $v$. By our choice of $N$, this translate $\alpha^{\prime} \beta^{\prime}$ is null-homotopic in $\widetilde{X} \backslash C$. And it follows that its cyclic conjugate $\beta^{\prime} \alpha^{\prime}$ is likewise null-homotopic in $\tilde{X} \backslash C$.

Conjugation cells can be adjoined to show that $\beta$ is homotopic relative to its endpoints to a path of the form $\phi \beta^{\prime} \gamma^{-1}$ where $\phi$ is an $A$-path. See Figure 3.2 It follows that $\alpha \beta$ is homotopic, relative to its basepoint, in an enlarged subcomplex, to a loop $u=(\alpha \phi)\left(\beta^{\prime} \alpha^{\prime}\right)\left(\left(\alpha^{\prime}\right)^{-1} \gamma^{-1}\right)$. Observe that $u$ is of the form $\delta_{1} \rho \delta_{2}$ with $\rho$ null-homotopic in $\tilde{X} \backslash C$ and $\delta_{1} \delta_{2}$ an $A$-loop.

Doing this for each type 2 generator yields an admissible subcomplex $C_{1}$ where $\pi_{1}\left(C_{1}\right)$ has a generating set with each type 2 generator having the desired form.

It is perhaps worth noting that at this point if $Y$ (that is, $H$ ) was known to be qsf then it would be easy to finish up the proof that $X$ is qsf. The argument involves 


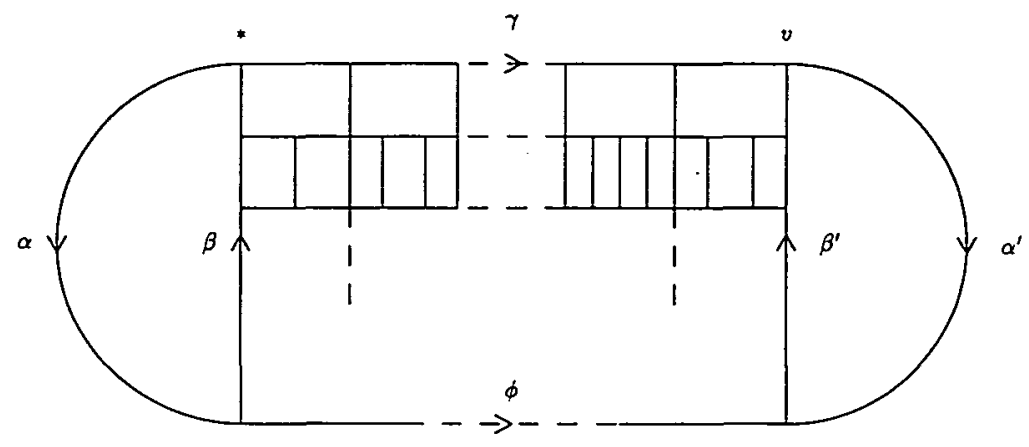

Figure 3.2

replacing each non-empty $C_{1} \cap l \tilde{Y}$ with a simply connected finite complex that maps into $l \tilde{Y}$, as well as attaching disks to each of the loops $\rho$ and mapping them into $\widetilde{X} \backslash C$. We leave any further details to the reader.

\section{FINISHING UP}

At this point we have an admissible subcomplex $C_{1}$ containing $C$ with $\pi_{1}\left(C_{1}\right)$ having a generating set of the form

$$
\left\{\tau_{1} \lambda_{1} \tau_{1}^{-1}, \ldots, \tau_{s} \lambda_{s} \tau_{s}^{-1}, \delta_{11} \rho_{1} \delta_{21}, \ldots, \delta_{1 t} \rho_{t} \delta_{2 t}\right\}
$$

where the $\lambda_{i}$ 's and the $\delta_{1 j} \delta_{2 j}$ 's are $A$-loops and the $\rho_{j}$ 's are null-homotopic in $\tilde{X} \backslash C$. In this section, we shall adjoin conjugation cells and attach a $B$-edge so as to obtain a subcomplex $K$ containing $C$ such that $\pi_{1}(K)$ has a generating set of the form

$$
\left\{\tau_{1} \lambda_{1} \tau_{1}^{-1}, \ldots, \tau_{s} \lambda_{s} \tau_{s}^{-1}, \ldots, \tau_{r} \lambda_{r} \tau_{r}^{-1}, \delta_{11} \rho_{1} \delta_{21}, \ldots, \delta_{1 t} \rho_{t} \delta_{2 t}\right\}
$$

where all of the $A$-loops $\lambda_{i}$ and $\delta_{1 j} \delta_{2 j}$ are freely homotopic in $K$ to $A$-loops $\left\{u_{k}\right\}$ that themselves are null-homotopic in $\widetilde{X} \backslash C$. As remarked in $\$ 2$ above, we can attach disks to each loop $u_{k}$ and to each $\rho_{j}$, yielding a simply connected finite complex $D$, and map each of the disks into $\tilde{X} \backslash C$. This will finish the proof that $X$ is qsf.

Since $C_{1}$ is a finite subcomplex, there are only finitely many components $l \widetilde{Y}$ that meet $C_{1}$. As the group $L$ is infinite, there is a $B$-edge $b$ with initial vertex $v_{1} \in C_{1}$ and terminal vertex $v_{2}$ in some component $l \tilde{Y}$ that does not meet $C_{1}$. Consider the subcomplex $C_{1} \cup b$. Clearly $\pi_{1}\left(C_{1}\right)=\pi_{1}\left(C_{1} \cup b\right)$ and $C_{1} \cup b$ is admissible.

Define a finite graph $\Gamma$ as follows: the vertices of $\Gamma$ are those components $l \widetilde{Y}$ that meet $C_{1} \cup b$, the edges of $\Gamma$ are the $B$-edges of $C_{1} \cup b$. Let $\bar{v}_{2}$ be the vertex that contains $v_{2}$. 
We shall be considering subcomplexes $K$ obtained from $C_{1} \cup b$ by adjoining conjugation cells. Given such a $K$ and given a subtree $\mathcal{T}$ of $\Gamma$ containing the vertex $\bar{v}_{2}$, we shall say that its $A$-loops are carried by $\mathcal{T}$ if $\pi_{1}(K)$ has a set of generators of the form

$$
\left\{\tau_{1} \lambda_{1} \tau_{1}^{-1}, \ldots, \tau_{s} \lambda_{s} \tau_{s}^{-1}, \ldots, \tau_{r} \lambda_{r} \tau_{r}^{-1}, \delta_{11} \rho_{1} \delta_{21}, \ldots, \delta_{1 t} \rho_{t} \delta_{2 t}\right\}
$$

where all of the $A$-loops $\lambda_{i}$ and $\delta_{1 j} \delta_{2 j}$ are freely homotopic in $K$ to $A$-loops that are in $U T^{(0)}$ (recall that the vertices are components $l \widetilde{Y}$ ). Formally, we shall be working with 4-tuples $(K, \sigma, \mathcal{T}, \mathcal{S})$ where $\sigma$ is the set of generators for $\pi_{1}(K)$ of the above form and $\mathcal{S}$ is a set of $A$-loops in $U T^{(0)}$ such that each of the $A$-loops $\lambda_{i}$ and $\delta_{1 j} \delta_{2 j}$ are freely homotopic in $K$ to some loop in $\mathcal{S}$. Call such 4-tuples acceptable.

Letting $K=C_{1} \cup b$, we see that its $A$-loops are carried by a maximal tree of $\Gamma$. Thus there is an acceptable 4-tuple. We shall use induction on the size of the subtrees $T$ to prove that there is some such $K$ with its $A$-loops carried by the singleton $\left\{\bar{v}_{2}\right\}$, that is, an acceptable 4-tuple $\left(K, \sigma,\left\{\bar{v}_{2}\right\}, \mathcal{S}\right)$.

Suppose $(K, \sigma, T, \mathcal{S})$ is an acceptable 4 -tuple. Since $\mathcal{T}$ is finite, there is at least one vertex different from $\bar{v}_{2}$ that is terminal in $\Gamma$, that is, has valency one. Let $\bar{v}$ be such a vertex, and let $\bar{b}$ be the $B$-edge in $\mathcal{T}$ emanating from $\bar{v}$. And let $\bar{v}^{\prime}$ be the terminal vertex of $\bar{b}$ in $\mathcal{T}$. We shall see how adjoining conjugation cells will yield a new acceptable 4-tuple with $A$-loops carried by $\mathcal{T} \backslash \bar{b}$.

Assume $u$ is one of the $A$-loops in $\mathcal{S}$ which is contained in the vertex $\bar{v}$. Let $v$ be the initial vertex of $\bar{b}$ when viewed as an edge in $\widetilde{X}$. By the admissibility of $K$, there is an $A$-path $p$ in $K$ from $v$ to the basepoint of $u$. Consider the $A$-loop $p u p^{-1}=a_{1} \cdots a_{n}$. By adjoining conjugation cells as pictured in Figure 4.1, and applying lemma 2.2, we can easily homotope the loop $p u p^{-1}$ to an $A$-loop in $\vec{v}^{\prime}$. Note that this may create more type 1 generators of the form $\tau \lambda \tau^{-1}$, but the point is that, by lemma 2.2, each of the new $A$-loops, $\lambda$, is in $\bar{v}^{\prime}$. Doing so for each such $u$, yields the desired 4-tuple, $\left(K^{\prime}, \sigma^{\prime}, \mathcal{T} \backslash \bar{b}, \mathcal{S}^{\prime}\right)$.

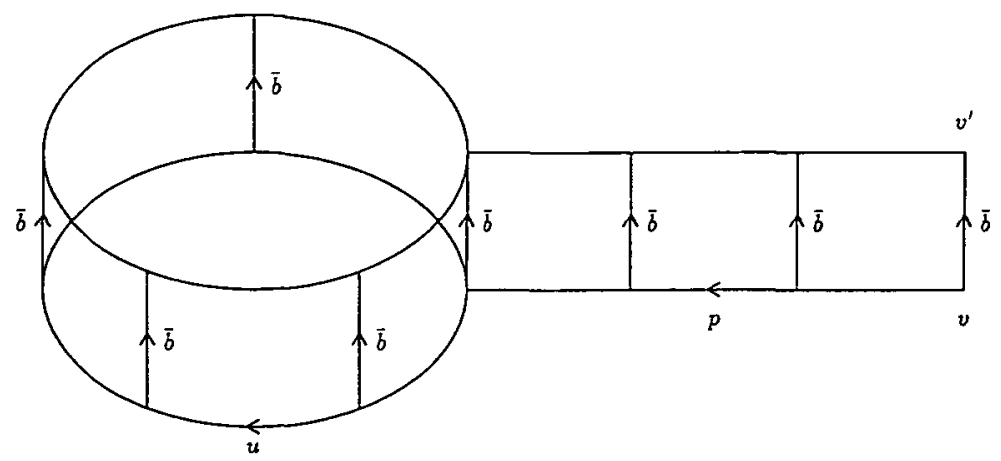

Figure 4.1 
Hence, by induction, there is an acceptable 4-tuple $\left(K, \sigma,\left\{\bar{v}_{2}\right\}, \mathcal{S}\right)$. Now each $A$ loop $u \in \mathcal{S}$ is in a copy $l \tilde{Y}$ of $\tilde{Y}$ disjoint from $C$ (the copy $l \tilde{Y}$ corresponds to the vertex $\bar{v}$ ). Since $l \tilde{Y}$ is simply connected, $u$ is null-homotopic in $l \tilde{Y} \subset \tilde{X} \backslash C$. As noted above, we can now conclude that $X$ is qsf.

\section{REFERENCES}

[1] S.G. Brick, 'Quasi-isometries and ends of groups', J. Pure Appl. Algebra (to appear).

[2] S.G. Brick and M.L. Mihalik, 'Qsf property for groups and spaces', (preprint).

[3] S.M. Gersten and J.R. Stallings, 'Casson's idea about 3-manifolds whose universal cover is $\mathbb{R}^{3}$, Internat. J. of Algebra and Comput. 1 (1991), 395-406.

[4] M.L. Mihalik and S.T. Tschantz, 'Tame combings and the quasi-simply-filtered condition for groups', (preprint).

[5] J. R. Stallings, 'Brick's quasi simple filtrations for groups and 3-manifolds', in Geometric methods in group theory, University of Sussex, July, 1991 (to appear).

Department of Mathematics

CSU-Fresno

Fresno CA 93740

United States of America

e-mail: stephen@zimmer.csufresno.edu
Department of Mathematics

Vanderbilt University

Nashville TN 37240

United States of America

e-mail: mihalikm@ctrvax.vanderbilt.edu 